\title{
Symmetry and inert states of spin Bose Condensates
}

\author{
S.-K. Yip \\ Insitute of Physics, Academia Sinica, Nankang 115, Taipei, Taiwan
}

(Dated: August 23, 2018)

\begin{abstract}
We construct the list of all possible inert states of spin Bose condensates for $S \leq 4$. In doing so, we also obtain their symmetry properties. These results are applied to classify line defects of these spin condensates in zero magnetic field.

PACS numbers: 03.75.Hh,03.75.Mn
\end{abstract}




\section{INTRODUCTION}

Optical trapping of atomic Bose gases [1, 2, 3, 4, 5] has provided us with exciting new physical systems, which have attracted significant theoretical interests (e.g. [6, 7, 8, 9, 10, 11, 12, 13]). In constrast to ${ }^{4} \mathrm{He}$, these Bosonic atoms possess finite hyperfine spins (hereafter simply as spins, denoted by $S$ ), hence internal degrees of freedom. Therefore in the Bose condensed phase, in contrast to ${ }^{4} \mathrm{He}$ where only the gauge symmetry is broken, the state can also have broken spin-rotational symmetries (we ignore the case of fragmented states [14] here). The present paper is devoted to symmetry considerations of these spincondensates. Knowing the broken symmetries, as well as the residual symmetries, is essential in understanding the properties of these spin superfluids, in particular topological excitations such as vortices.

The states that are expected to be realized are determined by minimization of (free) energy of the system. Such a program has been carried out by numerous authors [6, 7, 8, 12, 13], assuming that mean-field theory applies. The minimization of this free energy is rather simple [6, 7] for $S=1$, already not quite trivial [8] (see also [15]) for $S=2$, and indeed rather complicated [12, 13] for $S=3$. There are also a few attempts to find out the residual symmetries of these minimum energy states found [16, 17].

On the other hand, symmetry considerations can actually help in finding a special set of possible states known as "inert" states for the systems. These states are those where the wavefunction characterizing the state is actually independent of the (interaction) parameters that enter in the free energy that is being minimized. The form of these wavefunctions follow from symmetry considerations alone, depending only on the symmetries of the uncondensed (normal) state (thus independent of whether mean-field theory has been assumed). ¿From such considerations, the symmetries of these inert states are thus also directly determined. It is however, true that this procedure only determines the possible stationary points (relative minima, extrema or saddle points) of the free energy functional. Additional calculations (in particular the actual form of the free energy and the parameters which enter it) are needed to determine which state is actually a stable minima.

Such a program has actually been carried out before in other systems. In superfluid ${ }^{3} \mathrm{He}$, Cooper pairing occurs in angular momentum $L=1$ and spin $S=1$ channel. Spatial and/or spin rotational symmetries are in general broken in the superfluid states, and symmetry 
considerations [18, 19] (and references therein) have been used to find the inert states. These considerations have also been applied to "unconventional" superconductivity in solid state systems, where the superconducting state has symmetries lower than the underlying crystals, in particular in the context of heavy fermions, e.g., [20, 21]. In this case, the normal state possesses only discrete rather than continuous rotational symmetries.

In this paper, we shall carry out such a program for the spin condensates, finding the inert states and their symmetries. We shall limit ourselves to the case of no external magnetic field (linear and/or quadratic Zeeman fields). We discuss the general theory behind this work in Sec II]. Results up to $S=4$ are then presented in Sec III. We then apply these results to discuss vortices on Sec IV. Sec V provides a brief summary, and some details are relegated to the appendices.

\section{BASIC THEORY}

We here outline the basic theory behind this work. The present procedure of finding the inert states is based on a theorem by Michel [22]. This theorem has been discussed and explained in, e.g., [19]. Rather than restating this theorem in its full generality in abstract terms, we shall simply describe it physically for the case of $S=1$ here. Since $S=1$ has the same symmetry as angular momentum $L=1$, we shall use these two terminologies interchangably. In the uncondensed phase (the normal phase), the system is invariant under all spatial rotation $\mathrm{SO}(3)$ and gauge transformation $\mathrm{U}(1)$, and time reversal $\Theta$. (There is no need to consider inversion since we shall always consider fixed $L$, so all states have definite inversion symmetry). We are interested in finding the stationary points of a free energy $F[\Psi]$ which is a functional of $\Psi . F[\Psi]$ is invariant under any transformation $\Psi \rightarrow g \Psi$ where $g$ is any operation in the group $\mathrm{G}=\mathrm{SO}(3) \times \mathrm{U}(1) \times \Theta$. For $L=1$, it can be shown easily that all order parameters can be written in the form $\hat{k} \cdot(\hat{m}+c \hat{n}) e^{i \gamma}$ where $\hat{m}$ and $\hat{n}$ are real orthogonal unit vectors, and $c$ is a complex number with $|c| \leq 1$. (We shall, for simplicity of presentation, not always normalized our wavefunction, since this normalization has no effect on symmetries. We can restrict ourselves to $|c| \leq 1$ because if otherwise, we can always divide the state by $c$ and then rewrite the state in the above form). It is also sufficient to consider only the two cases $c=0$ and $\operatorname{Im} c \neq 0$ (if $\operatorname{Im} c=0$, then the state is just $\left(\hat{k} \cdot \hat{m}^{\prime}\right) e^{i \gamma}$ for some $\hat{m}^{\prime}$ ). Special choices of $\hat{m}, \hat{n}$ and phase factor $\gamma$ results in reference states, which 
we shall take to be $\hat{k} \cdot \hat{z}$ and $\hat{k} \cdot(\hat{x}+c \hat{y})$. All states $(\hat{k} \cdot \hat{m}) e^{i \gamma}$ can be obtained from the reference state $\hat{k} \cdot \hat{z}$ by rotation and gauge transformation. Equivalent statement applies to the states $\hat{k} \cdot(\hat{m}+c \hat{n}) e^{i \gamma}$ for a given $c$. [Technically, the collection of states $\{g \Psi\}$ obtained from $\Psi$ by operating the transformation $g$ on it are said to form the orbit of $\Psi]$. As far as our problem of obtaining stationary states are concerned, these solutions related by symmetries are "equivalent" solutions. $\Psi$ is an inert state if and only if $g \Psi$ is an inert state.

An important consideration in determining whether a particular state $\Psi$ is an inert state is the symmetry group under which $\Psi$ is invariant, that is, the isotropy subgroup $\mathrm{H}$ of $\Psi$, where $\mathrm{H}$ contains all operations $h$ in $\mathrm{G}$ such that $h \Psi=\Psi$. We first note here that a state which can be obtained by a transformation $g$ on $\Psi$ has symmetry simply related to that of the original reference state $\Psi$. [Technically, $g \Psi$ has the isotropy group $g H^{-1}$ thus these isotropy groups are said to be conjugate to each other]. Consider now the reference state $\Psi_{c}=\hat{k} \cdot(\hat{x}+c \hat{y})$. If $c=c_{1}+i c_{2}$ with $c_{1} \neq 0$ and $c_{2} \neq 0$ then $\mathrm{H}$ contains the identity $E$ alone. All states with $c_{1} \neq 0$ and $c_{2} \neq 0$ have the same symmetries. [Technically, the collection of orbits $\left\{g \Psi_{c}\right\}$ from states $\Psi_{c}$ with the same symmetry are said to form a stratum]. For any given $\left(c_{1}, c_{2}\right) \neq(0,0)$, we can choose a $\left(c_{1}^{\prime}, c_{2}^{\prime}\right)$ arbitrary close to the original $\left(c_{1}, c_{2}\right)$ such that $\Psi_{c^{\prime}}\left(\neq g \Psi_{c}\right.$ for any $g$ in $\left.\mathrm{G}\right)$ has the same symmetry as the original $\Psi_{c}$. [Technically, the orbit $\left\{g \Psi_{c}\right\}$ therefore not isolated in its stratum]. Michel's theorem then says that the state $\Psi_{c}$ is not an inert state. For example, we do not expect the state $\hat{k} \cdot\left(\hat{x}+\frac{1+i}{\sqrt{3}} \hat{y}\right)$ with $c=\frac{1+i}{\sqrt{3}}$ to be an inert state. While it may minimize a particular free energy $F$, there is no reason to expect $c$ to remain at the same value of $c=\frac{1+i}{\sqrt{3}}$ when the parameters in the free energy $F$ are changed. Similar remarks apply to the case where $c=i c_{2}$ with $\left|c_{2}\right|<1$ (though $\mathrm{H}$ contains also other elements in additional to E).

The states with, however, $c=i$ is special in that the isotropy group $\mathrm{H}$ would then become $\left\{R_{z}(\alpha) e^{-i \alpha}, U_{2}^{\gamma} e^{-2 i \gamma} \Theta\right\}$ where $R_{z}(\alpha)$ denotes a rotation of angle $\alpha$ about $\hat{z}$, and $U_{2}^{\gamma}$ denotes a $\pi$ rotation about the horizontal axis $\hat{\gamma}=\cos \gamma \hat{x}+\sin \gamma \hat{y}$. (Note that $\Theta$ does not commute with $e^{-i 2 \gamma}$. We are using the notation where operations start from the left). A small modification of $c$ away from $i$ would result in a state that has a completely different symmetry (and is not $g \Psi$ for some $g$ in $\mathrm{G}$, that is, not simply another order parameter related to $\Psi$ by a transformation in G). [Technically, we say that the orbit $g \Psi$ is isolated in its stratum]. Michel's theorem then states that $\Psi_{c=i}$ is an inert state. This state is a stationary point of the free energy irrespective of the parameters that enter in $F$. 
Similar statement applies to $\Psi=\hat{k} \cdot \hat{z}$, (related to the state $\Psi_{c=0}$ by a rotation) where the isotropy group is $\mathrm{H}=\left\{R_{z}(\alpha), U_{2}^{\gamma} e^{i \pi}\right\} \times \Theta$. (From the above argument, we have in fact shown that the only inert states for $\mathrm{L}=1$ are the axial state $\hat{k} \cdot(\hat{x}+i \hat{y})$ and the polar state $\hat{k} \cdot \hat{z}$. In the spin wavefunction language they are $(1,0,0)$ and $(0,1,0)$ respectively, with isotropy groups already given above).

Therefore, in general (not restricted to $L=1$ ), one is searching for states $\Psi$ that have special symmetries in the sense that a slight deviation of the coefficients entering $\Psi$ would give another state $\Psi^{\prime}$ with an isotropy group different from $\mathrm{H}$ (but barring cases where $\Psi^{\prime}$ is just an "equivalent" solution, that is, $\Psi^{\prime}=g \Psi$ and thus just a symmetry-transformed $\Psi$, i.e., cases where the new order parameter is in the same orbit and thus with symmetry group $g \mathrm{Hg}^{-1}$ ) [Technically, we look for states $\Psi$ such that its orbit is isolated in its stratum].

The procedure is thus to search for all subgroups $\mathrm{H}$ of the combined group $\mathrm{SO}(3) \times \mathrm{U}(1)$ $\times \Theta$ with broken $\mathrm{U}(1)$ gauge symmetry which would determine $\Psi$ uniquely. Each of these $\Psi$ would then be an inert state. (In principle it is sufficient that $\mathrm{H}$ determines a set of possible $\Psi$ 's, such that the orbits of these order parameters are isolated. However, in the present case of interest, this ambiguity is only in an overall \pm sign for the wavefunction. Thus effectively each of these $\mathrm{H}$ determines a unique inert state $\Psi$.) It is useful to note that once such an $\mathrm{H}$ is found, then there is no need to consider the subgroups of $\mathrm{H}$. This is because any $\Psi_{2}$ invariant under a subgroup $\mathrm{H}_{2}$ of $\mathrm{H}_{1}$ but not $\mathrm{H}_{1}$ itself cannot yield an inert state. (To see this, consider $\Psi(\lambda)=\Psi_{2}+\lambda \Psi_{1}$ where $\Psi_{1}\left(\neq \Psi_{2}\right)$ is invariant under $\mathrm{H}_{1}$. Then $\Psi(\lambda)$ is invariant under $\mathrm{H}_{2}$ for any $\lambda$ and hence, by the argument given, $\Psi_{2}$ cannot be an inert state.)

The subgroups of $\mathrm{SO}(3) \times \mathrm{U}(1) \times \Theta$ that break the gauge symmetry can be constructed in a similar manner as in [20]. There, Volovik and Gorkov constructed all possible isotropy subgroups for unconventional superconductors where the normal state possesses discrete rotational symmetries of crystals instead of $\mathrm{SO}(3)$. A principal observation, which still applies here, is that $\mathrm{U}(1)$ is isomorphic to rotation about a fixed axis. Thus the required groups would involve elements that are in general product of a spatial rotation and a gauge transformation.

In our present case, these subgroups fall into two categories. The first involves continuous groups. These can be constructed easily (see below). The second involves discrete groups. Our task is simplified since the discrete subgroups of $\mathrm{SO}(3)$ are well known. They are the 
three groups of regular polyhedrons: $\mathrm{O}$ of the octahedron, $\mathrm{T}$ the tetrahedron, and $\mathrm{Y}$ the icosahedron; the dihedral groups $\mathrm{D}_{m}$ and the cyclic groups $C_{m}$.

Before we actually list these groups we note further a great simplication. Let us consider a general spin wavefunction $\Psi=\left(\zeta_{S}, \ldots, \zeta_{-S}\right)$. If the group $\mathrm{H}$ contains the symmetry element $h=C_{n} e^{i j 2 \pi / n}$ where $j$ is an integer which can be restricted to $-n<j<n$ ( $j$ is an integer follows from the requirement that $h^{n}$ must also be in $\mathrm{H}$ : as $C_{n}^{n}=1$, we must have $j$ being an integer), since under $C_{n}, \zeta_{m} \rightarrow \zeta_{m} e^{-i m 2 \pi / n}$, we must have $e^{i(j-m) 2 \pi / n}=1$ for $\zeta_{m} \neq 0$. Hence only $\zeta_{j}, \zeta_{j \pm n}$ etc can be finite. If there is only one such finite $\zeta_{m}$ then the order parameter has been determined uniquely. The state then has in fact continuous symmetry $R_{z}(\alpha) e^{i j \alpha}$. If there are more than one non-zero $\zeta_{m}$, say $\zeta_{j}$ and $\zeta_{j-n}$, then it can be an inert state only if there are further other symmetry elements in $\mathrm{H}$ that puts a constraint relating $\zeta_{j}$ and $\zeta_{j-n}$. Only when $j-n=j$ can this be achieved by a horizontal rotational axis, and the symmetry group would then be a combined group constructed from the dihedral groups $\mathrm{D}_{n}$ (or a larger group containing this). If $j-n \neq j$, such requirements would arise only from groups that are constructed from the regular-polyhedral groups $\mathrm{O}, \mathrm{T}$ and $\mathrm{Y}$.

Hence we can follow the following procedure. First we consider all the continuous isotropy subgroups and find the corresponding order parameters. Then we consider the (non-dihedral) subgroups that are constructed from the polyhedral groups: $\mathrm{O} \times \mathrm{U}(1) \times \Theta, \mathrm{T} \times \mathrm{U}(1) \times \Theta$, and $\mathrm{Y} \times \mathrm{U}(1) \times \Theta$. If an order parameter for a particular isotropy group can be found, then we discard all its subgroups. If not, then we go to a group of lower symmetry. This is basically the only part of the procedure that is more involved. After such groups are exhausted, the only inert states that can be left are those correspond to the dihedral symmetries. These states can be obtained by inspection (with the procedure already hinted above in the last paragraph: see examples below). For our case with $L \leq 4$, we shall argue in Appendix A that five-fold symmetry elements cannot be involved, hence there is no need to consider the icosahedral group Y (and also dihedral groups containing $C_{5}$ ). The only non-dihedral polyhedral groups needed are thus those constructed from $\mathrm{O} \times \mathrm{U}(1) \times \Theta$ and $\mathrm{T} \times \mathrm{U}(1) \times \Theta$, and they can already be found in [20].

Now we proceed therefore to list the continuous subgroups, and subgroups which can be constructed from the polyhedral groups $\mathrm{O}$ and $\mathrm{T}$. The continuous ones are easy. We can combine $R_{z}(\alpha)$, continuous rotation of angle $\alpha$ about the axis $z$, with gauge transformation $e^{i n \alpha}$ where $n$ is an integer. The only state that would be invariant under $R_{z}(\alpha) e^{i n \alpha}$ for all 
$\alpha$ is the wavefunction where the only non-zero component $\zeta_{m}$ in $\Psi$ is $\zeta_{n}$. Corresponding wavefunction of angular momentum $L$ is $\zeta_{n} Y_{L}^{n}(\hat{k})$. This wavefunction automatically has symmetry elements around horizontal axes $\hat{\gamma}$ combined with suitable phase factors (due to the definition from spherical harmonics). For $L-n$ even, the isotropy group is

$$
\mathrm{H}_{\mathrm{n}}^{\mathrm{e}} \equiv\left\{R_{z}(\alpha) e^{i n \alpha}, U_{2}^{\gamma} e^{2 i n \gamma} \Theta\right\}
$$

whereas for $L-n$ odd, we have

$$
\mathrm{H}_{\mathrm{n}}^{\mathrm{o}} \equiv\left\{R_{z}(\alpha) e^{i n \alpha}, U_{2}^{\gamma} e^{i(2 n \gamma+\pi)} \Theta\right\}
$$

We need only consider non-negative $n$ 's, since the state with only $\zeta_{-n}$ being non-zero is related to the state with $\zeta_{n}$ being the only non-zero element via either the time-reversal symmetry or a horizontal rotation by $\pi$.

For the discrete (non-dihedral) subgroups contructed from $\mathrm{O}$ and $\mathrm{T}$, they have already been considered by Volovik and Gorkov [20] and so we simply list their answers below:

$$
\mathrm{O} \times \Theta \equiv\left\{E, 8 C_{3}, 3 C_{2}, 6 U_{2}, 6 C_{4}\right\} \times \Theta
$$

This group corresponds to just breaking the gauge symmetry in $\mathrm{O} \times \mathrm{U}(1) \times \Theta$. Here $C_{3}$ are three-fold rotations along the diagonals, $U_{2}$ are two-fold rotations along the edges, and $C_{4}$ are four-fold rotations about the faces of a cube, and $C_{2}=C_{4}^{2}$.

$$
\mathrm{O}(\mathrm{T}) \times \Theta \equiv\left\{E, 8 C_{3}, 3 C_{2}, 6 U_{2} e^{i \pi}, 6 C_{4} e^{i \pi}\right\} \times \Theta
$$

This group is isomorphic to $\mathrm{O}$, but contains only $\mathrm{T}$ as a subgroup.

$$
\begin{aligned}
\mathrm{O}\left(\mathrm{D}_{2}\right) \equiv & \left\{E, 3 C_{2}, 2 C_{4}^{x} \Theta, 2 C_{4}^{y} \epsilon \Theta, 2 C_{4}^{z} \epsilon^{2} \Theta\right. \\
& \left.4 C_{3} \epsilon, 4 C_{3}^{2} \epsilon^{2}, 2 U_{2}^{\perp x} \Theta, 2 U_{2}^{\perp y} \epsilon \Theta, 2 U_{2}^{\perp z} \epsilon^{2} \Theta\right\}
\end{aligned}
$$

This group contains $D_{2}$ as a subgroup. Here $U_{2}^{\perp x}$ denotes a $\pi$ rotation about the edge of a cube perpendicular to $x$ (i.e., $x \rightarrow-x$ and $y \leftrightarrow z$ or $y \leftrightarrow-z$ ), and $\epsilon \equiv e^{2 i \pi / 3}$.

We also have the tetrahedral groups

$$
\mathrm{T} \times \Theta \equiv\left\{E, 3 C_{2}, 4 C_{3}, 4 C_{3}^{2}\right\} \times \Theta
$$


and

$$
\mathrm{T}\left(\mathrm{D}_{2}\right) \equiv\left\{E, 3 C_{2}, 4 C_{3} \epsilon, 4 C_{3}^{2} \epsilon^{2}\right\}
$$

This last group is isomorphic to $\mathrm{T}$ but only contains $\mathrm{D}_{2}$ as a subgroup. We note here also that $\mathrm{T}$ is a subgroup of both $\mathrm{O}$ and $\mathrm{O}(\mathrm{T})$ and $\mathrm{T}\left(\mathrm{D}_{2}\right)$ is a subgroup of $\mathrm{O}\left(\mathrm{D}_{2}\right)$. It turns out that neither $\mathrm{T}$ nor $\mathrm{T}\left(\mathrm{D}_{2}\right)$ give rise to inert states for any of the cases we have examined below.

This complete the list of all non-dihedral groups that we would need (for $L \leq 4$ ).

\section{RESULTS}

Now we apply our theory to, in order $S($ or $L)=2,3,4$. For simplicity in notations, when writing the wavefunction for $L$, we would simply write $x, y, z$ instead of $\hat{k}_{x}, \hat{k}_{y}, \hat{k}_{z}$. It is understood that $x^{2}+y^{2}+z^{2}=1$.

\section{A. $S=2$}

The inert states for $L=2$ corresponding to d-wave Cooper pairing has in fact been investigated before by [18] (see also [19]) by considering the system as strongly spin-orbit coupled $L=1$ and $S=1$ Cooper pairs with total angular momentum $J=2$. Here we would like to present our arguments directly in terms of the procedure outline above so that it can be easily compared with the results for $S=3$ and 4 .

Continuous Groups: We can easily write down the three order parameters corresponding to continuous groups $\mathrm{H}_{\mathrm{n}}^{\mathrm{e}, \mathrm{o}}$ for $n=2,1,0$. We shall call these states $\mathrm{F}_{2}, \mathrm{~F}_{1}$ and $\mathrm{P}_{0}$ (Here $\mathrm{F}$

indicates ferromagnetic and $\mathrm{P}$ polar, using the language as in, e.g., [8, 23] ). Explicitly, the state wavefunctions are

$$
\begin{aligned}
& \mathrm{F}_{2} \equiv(1,0,0,0,0) \\
& \mathrm{F}_{1} \equiv(0,1,0,0,0) \\
& \mathrm{P}_{0} \equiv(0,0,1,0,0)
\end{aligned}
$$

with corresponding polynomial forms $(x+i y)^{2},-z(x+i y)$ and $\left(2 z^{2}-x^{2}-y^{2}\right)$. 
Discrete groups: It can be easily verified that no order parameter can satisfy the groups $\mathrm{O} \times \Theta, \mathrm{O}(\mathrm{T}) \times \Theta$ and $\mathrm{T} \times \Theta$. To see this, we note that all $L=2$ wavefunctions can be written as polynomials of second degree in $x, y, z$. The elements $3 C_{2}$ require $\Psi$ to be a linear combination of $x^{2}, y^{2}, z^{2}$ only. (e.g., $x y$ is odd under $C_{2}^{x}$ and hence must be rejected). All these three groups contain eight three-fold rotations $C_{3}$ along the diagonal of a cube, where $(x, y, z) \rightarrow(y, z, x),(x, y, z) \rightarrow(-z,-x, y)$ etc. The only function that has this property is $x^{2}+y^{2}+z^{2}$, which however does not belong to $L=2$.

The discrete group $\mathrm{O}\left(\mathrm{D}_{2}\right)$ in eq (5) uniquely determines (up to a \pm sign) the order parameter to be $x^{2}+\epsilon y^{2}+\epsilon^{2} z^{2}$ (see also [20]). We have just seen that $3 C_{2}$ limit us to the polynomials $x^{2}, y^{2}$ and $z^{2}$. Under $C_{3}$, we have the mapping $(x y z) \rightarrow(y z x)$ and hence the elements $C_{3} \epsilon$ requires that the wavefunction be proportional to $x^{2}+\epsilon y^{2}+\epsilon^{2} z^{2}$. Elements such as $C_{4}^{x} \Theta$ fixes the overall phase factor to be real (under $\left.C_{4}^{x},(x, y, z) \rightarrow(x, z,-y)\right)$ ). We can also check that elements such as $U_{2}^{\perp x} \Theta$ are satisfied. As before [8, 23], we shall refer to this state as the "cyclic" state. We shall denote it by C, and further discuss this state below.

Since we have already found the order parameter for $\mathrm{O}\left(\mathrm{D}_{2}\right)$, there is no need to consider its subgroup $\mathrm{T}\left(\mathrm{D}_{2}\right)$. This completes the non-dihedral polyhedral groups.

It remains then to consider the ones corresponding to order parameters where only $\zeta_{ \pm m} \neq$ 0 for some $m \neq 0$ and symmetries related to the dihedral groups. For $m=2$ we can have the state

$$
\mathrm{P}_{2} \equiv(1,0,0,0,1)
$$

Its polynomial form is $x^{2}-y^{2}$ and easily seen to possess the symmetry (same notation as in $[20]$ )

$$
\mathrm{D}_{4}\left(\mathrm{D}_{2}\right) \times \Theta \equiv\left\{E, C_{2}, 2 U_{2}, C_{4} e^{i \pi}, 2 U_{2}^{\prime} e^{i \pi}\right\} \times \Theta
$$

It can easily be checked that this group actually determines the order parameter uniquely (again up to \pm sign, a warning that we would not repeat again below) to be $x^{2}-y^{2}$, and hence the state is inert. Note that a rotational about $z$ changes the relative phase between $\zeta_{ \pm 2}$, thus all states of the form $e^{i \phi}\left(e^{-2 i \alpha}, 0,0,0, e^{2 i \alpha}\right)$ are related to $\mathrm{P}_{2}$ by operations within G. States with $\left|\zeta_{-2}\right| \neq\left|\zeta_{2}\right|$ as the only non-zero elements have lower symmetries (in the sense of subgroup) and therefore need not be considered. 
One may attempt to try the state $\Psi=(0,1,0,1,0)$, which in polynomial form reduces to $-i 2 z y$. Thus up to a gauge tranformation and a rotation, this state is just the same as $2 x y$. We note here that a $\pi / 4$ rotation about $z$ changes $x^{2}-y^{2}$ to $2 x y$. Thus the state $(0,1,0,1,0)$ can be transformed to the state $\mathrm{P}_{2}$ and does not correspond to a new inert state.

This completes our search for inert states for $L=2$. We found five inert states: $\mathrm{F}_{2}, F_{1}$, $\mathrm{P}_{0}$ with continuous symmetries, $\mathrm{C}$ and $\mathrm{P}_{2}$ with discrete symmetries.

In previous works [8, 15], actual minimization of general quartic free energy were performed. Our inert states accounted for all minina found in [8, 15]. There, it turns out that $\mathrm{F}_{1}$ is never the absolute minimum for any parameters entering $F[\Psi]$. It also turns out to be a peculiar fact that, to this order, $\mathrm{P}_{0}$ and $\mathrm{P}_{2}$ are always degenerate. Furthermore, any real linear combination between $\mathrm{P}_{0}$ and $\mathrm{P}_{2}$ are also degenerate [15] [Mermin has shown that, when the state is written in the form $B_{i j} x_{i} x_{j}$ where $x_{1,2,3}=x, y, z$, any real symmetric traceless $B_{i j}$ produces the same free energy. Since any such matrix $B_{i j}$ can be diagonalized, we see that these $\Psi$ can always be recast into the form $a\left(2 z^{2}-x^{2}-y^{2}\right)+b\left(x^{2}-y^{2}\right)$ with $a$ and $b$ real. This is thus a real linear combination of $\mathrm{P}_{0}$ and $\left.\mathrm{P}_{2}\right]$ We however expect that these accidental degeneracies would be lifted in more general free energies (e.g. containing higher order terms in $\Psi)$.

Let us further comment on the cyclic state. It can easily be checked that the polynomial $\epsilon x^{2}+\epsilon^{2} y^{2}+z^{2}$ is proportional to $\sqrt{2} Y_{2}^{0}+i\left(Y_{2}^{2}+Y_{2}^{-2}\right)$, and hence the state vector can be written as

$$
\mathrm{C} \equiv(i, 0, \sqrt{2}, 0, i)
$$

corresponding to a form used in [8]. A $\pi / 4$ rotation around $\hat{z}$ can transform this into $(1,0, \sqrt{2}, 0,-1)$, that is $\sqrt{2} Y_{2}^{0}+\left(Y_{2}^{2}-Y_{2}^{-2}\right)$. We remark here that this state was in fact found by Anderson and Morel [24] to be the minimum energy state for a d-wave Fermi superfluid within weak-coupling BCS theory. A pictorial way of showing the symmetry of this state can also be found there (see also [20]).

Since the group $\mathrm{O}\left(\mathrm{D}_{2}\right)$ has explicitly the symmetry element $C_{3} \epsilon$, we know immediately that, if one uses quantization axis along this axis, the wavefunction must assume the form $\left(0, \zeta_{1}, 0,0, \zeta_{-2}\right)$, i.e., only $\zeta_{1}$ and $\zeta_{-2}$ are non-zero. Indeed, let us take $\hat{w} \equiv(\hat{x}+\hat{y}+\hat{z}) / \sqrt{3}$ and $\hat{u} \equiv(-\hat{x}+\hat{y}) / \sqrt{2}$, and $\hat{v} \equiv \hat{w} \times \hat{u}$ to be the new orthonormal axes. Using the transformation from $\vec{r}=x \hat{x}+y \hat{y}+z \hat{z}$ to the new coordinates $\vec{r}=u \hat{u}+v \hat{v}+w \hat{w}$, simple algebra shows that $\epsilon x^{2}+\epsilon^{2} y^{2}+z^{2}=-\sqrt{2} i w(u+i v)-\frac{(u-i v)^{2}}{2}$. Therefore, with $\hat{w}$ as the quantization 
axis, the wavefunction can then be also written as $(0, \sqrt{2} i, 0,0,-1)$, the form as advertized. Of course, there are also many other possible forms for $\mathrm{C}$ (see., e.g., [10]). We remind the reader here that this state is unique up to gauge and rotations (as also proven by Mermin [15]).

\section{B. $S=3$}

The states that have continuous symmetries are

$$
\begin{aligned}
& \mathrm{F}_{3} \equiv(1,0,0,0,0,0,0) \\
& \mathrm{F}_{2} \equiv(0,1,0,0,0,0,0) \\
& \mathrm{F}_{1} \equiv(0,0,1,0,0,0,0) \\
& \mathrm{P}_{0} \equiv(0,0,0,1,0,0,0)
\end{aligned}
$$

with corresponding polynomial forms $-(x+i y)^{3}, z(x+i y)^{2},-\left(5 z^{2}-1\right)(x+i y)$, and $\left(5 z^{3}-3 z\right)$.

To construct the states belonging to the polyhedral groups, it is convenient to first notice that an $L=3$ state can always be written in the form of a cubic polynomial in $x, y, z$, thus $B_{i j k} x_{i} x_{j} x_{k}$ where $x_{1,2,3} \equiv x, y, z$. We can further limit ourselves to $B_{i j k}$ symmetric under all possible interchanges of indices $i j k$. Orthogonality to $L=1$ then requires < $\left.B_{i j k} x_{i} x_{j} x_{k} x_{l}\right\rangle=0$ where $\left\langle\ldots>\right.$ indicates an angular average. Hence we have $\sum_{i} B_{l i i}=0$ and so it is sufficient to specify only seven $(=2 \times 3+1)$ coefficients, which can be chosen to be $B_{x y z}, B_{x y y}, B_{x z z}, B_{y x x}, B_{y z z}, B_{z x x}, B_{z y y}$ (by eliminating e.g., $B_{x x x}$ in terms of $B_{x y y}$ and $\left.B_{x z z}.\right)$ All the polyhedral groups $\mathrm{O} \times \Theta, \mathrm{O}(\mathrm{T}) \times \Theta, \mathrm{O}\left(\mathrm{D}_{2}\right), \mathrm{T} \times \Theta$ and $\mathrm{T}\left(\mathrm{D}_{2}\right)$, contain $3 C_{2}$. The only polynomial that satisfies this is $x y z$. (e.g., invariance under $C_{z}^{x}$ leaves only $x y z, x y y$ and $x z z$. The latter two are eliminated by the requirement of invariance under $C_{2}^{z}$.) Therefore obviously $\mathrm{O}, \mathrm{O}\left(\mathrm{D}_{2}\right), \mathrm{T}\left(\mathrm{D}_{2}\right)$ cannot be satisfied. However, it does satisfy $\mathrm{O}(\mathrm{T}) \times \Theta$. We can check in fact $\mathrm{O}(\mathrm{T}) \times \Theta$ uniquely determines the order parameter to be $x y z$. Note that $x y z=z \operatorname{Im}(x+i y)^{2}$, hence the spin-wavefunction for $x y z$ can be written as $(0,-i, 0,0,0, i, 0)$. Though by a suitable rotation about $z$, we can change this to the form $(0,1,0,0,0,1,0)$ and thus call this $\mathrm{P}_{2}$, for reasons to be explained below we shall instead call this state D: 


$$
\mathrm{D} \equiv(0,-i, 0,0,0, i, 0)
$$

Since $\mathrm{T} \times \Theta$ is a subgroup of $\mathrm{O}(\mathrm{T}) \times \Theta$, we have exhausted all states under the nondihedral polyhedral groups. Hence we proceed to the states with only $\zeta_{ \pm m}$ being finite obeying dihedral groups. For $m=3$ we can have the state $(-1,0,0,0,0,0,1)$, and we shall call this $\mathrm{P}_{3}$. It has the polynomial form $\operatorname{Re}(x+i y)^{3}$ and the isotropy group

$$
\mathrm{D}_{6}\left(\mathrm{D}_{3}\right) \times \Theta \equiv\left\{E, 2 C_{3}, 3 U_{2}, C_{2} e^{i \pi}, 2 C_{6} e^{i \pi}, 3 U_{2}^{\prime} e^{i \pi}\right\} \times \Theta .
$$

We can check again that $\mathrm{D}_{6}\left(\mathrm{D}_{3}\right)$ uniquely determines the order parameter to be $\operatorname{Re}(x+i y)^{3}$. For $m=2$, we would obtain the state $\mathrm{P}_{2}$ which was already discussed under the group $\mathrm{O}(\mathrm{T})$ $\times \Theta$. For $m=1$, the wavefunction $(0,0,0,1,0,1,0,0,0)$ would then have the form $\left(5 z^{2}-1\right) y$ up to a phase factor $i$. This state is equivalent to $\left(5 z^{2}-1\right) x$ which has the symmetry $\mathrm{D}_{2}\left(\mathrm{U}_{2}\right) \times \Theta=\left\{E, C_{2}^{z} e^{i \pi}, U_{2}, U_{2}^{\prime} e^{i \pi}\right\} \times \Theta$ and is thus a subgroup of $\mathrm{D}_{6}\left(\mathrm{D}_{3}\right) \times \Theta$ considered before and thus this state is not an inert state. We thus have exhausted the list for states with dihedral symmetries.

Summarizing, for $S=3$ we found six inert states, $\mathrm{F}_{3}, \mathrm{~F}_{2}, \mathrm{~F}_{1}$ and $\mathrm{P}_{0}$ with continuous symmetries, $\mathrm{D}$ and $\mathrm{P}_{3}$ with discrete symmetries.

Minimization of a general quartic free energy has been carried out by [12, 13]. In [12], $\mathrm{F}_{3}, \mathrm{~F}_{2}$ and $\mathrm{P}_{3}$ appeared explicitly as absolute minima for suitable interaction parameters. They also find a state (which they named state $\mathrm{D}$ ) which we shall call $\mathrm{D}^{\prime}$ with the spin wavefunction $(\sqrt{2}, 0,0, i \sqrt{5}, 0,0, \sqrt{2})$. The form suggests that it is an inert state. Since it has three-fold symmetry but it is obviously not $\mathrm{P}_{3}$, this suggests that it should be the state $\mathrm{D}$ expressed in rotated axis. We shall verify that this is indeed the case in Appendix B, Thus their state $\mathrm{D}^{\prime}$ has isotropy group (conjugate to) $\mathrm{O}(\mathrm{T}) \times \Theta$. 
C. $S=4$

For continuous groups, we have

$$
\begin{aligned}
& \mathrm{F}_{4} \equiv(1,0,0,0,0,0,0,0,0) \\
& \mathrm{F}_{3} \equiv(0,1,0,0,0,0,0,0,0) \\
& \mathrm{F}_{2} \equiv(0,0,1,0,0,0,0,0,0) \\
& \mathrm{F}_{1} \equiv(0,0,0,1,0,0,0,0,0) \\
& \mathrm{P}_{0} \equiv(0,0,0,0,1,0,0,0,0)
\end{aligned}
$$

with polynomial forms $(x+i y)^{4},-z(x+i y)^{3},\left(7 z^{2}-1\right)(x+i y)^{2},-\left(7 z^{3}-3 z\right)(x+i y)$, $\left(35 z^{4}-30 z^{2}+3\right)$. For discrete groups, we have to consider even order polynomials up to fourth order (we shall require orthogonality to $L=0$ and 2 later). For the polyhedral groups $\mathrm{O} \times \Theta, \mathrm{O}(\mathrm{T}) \times \Theta, \mathrm{O}\left(\mathrm{D}_{2}\right), \mathrm{T} \times \Theta$ and $\mathrm{T}\left(\mathrm{D}_{2}\right)$, the elements $3 C_{2}$ eliminated all polynomials except $x^{4}, y^{4}, z^{4}, y^{2} z^{2}, z^{2} x^{2}, x^{2} y^{2}, x^{2}, y^{2}, z^{2}$. To obey $8 C_{3}$ in $\mathrm{O} \times \Theta$ and $\mathrm{O}(\mathrm{T}) \times \Theta$, we must then have the linear combination

$$
\Psi=c\left(x^{4}+y^{4}+z^{4}\right)+a\left(y^{2} z^{2}+z^{2} x^{2}+x^{2} y^{2}\right)+b\left(x^{2}+y^{2}+z^{2}\right)
$$

Obviously $\mathrm{O}(\mathrm{T}) \times \Theta$ is not satisfied (no required sign changes under $C_{4}$ ), but $\mathrm{O} \times \Theta$ is if $c, a, b$ are all real. Before we continue, we note that the three functions multiplying the coefficients $c, a, b$ are not linearly independent. Indeed, using $x^{2}+y^{2}+z^{2}=1$ we can verify that $\left(x^{4}+y^{4}+z^{4}\right)=\left(x^{2}+y^{2}+z^{2}\right)-2\left(y^{2} z^{2}+z^{2} x^{2}+x^{2} y^{2}\right)$. Let us choose to use $\left(y^{2} z^{2}+z^{2} x^{2}+x^{2} y^{2}\right)$ and $x^{2}+y^{2}+z^{2}=1$ to be the two independent functions, thus setting $c=0$ in eq (17). Orthogonality to $L=0$ and 2 requires $<\Psi>=0,\left\langle\Psi x^{2}>=<\Psi y^{2}>=<\Psi z^{2}>=0\right.$. Evaluating the angular averages [29], we find that all these relations are satisfied if $b=-a / 5$. (The fact that these equations are dependent is not a coincidence, since $<x^{2}\left(x^{4}+y^{4}+z^{4}\right)>$ $/<\left(x^{4}+y^{4}+z^{4}\right)>=<x^{2}\left(y^{2} z^{2}+z^{2} x^{2}+x^{2} y^{2}\right)>/<\left(y^{2} z^{2}+z^{2} x^{2}+x^{2} y^{2}\right)>=<x^{2}>=1 / 3$ etc from symmetry.) Thus $\mathrm{O} \times \Theta$ determines the order parameter uniquely and the state is inert. Choosing $a=5$ gives us the wavefunction

$$
\Psi=5\left(y^{2} z^{2}+z^{2} x^{2}+x^{2} y^{2}\right)-1
$$


For lack of a better name we shall just call this state O. In Appendix C we shall verify that the corresponding spin wavefunction, up to a normalization factor and sign, is given by

$$
\mathrm{O} \equiv(\sqrt{5}, 0,0,0, \sqrt{14}, 0,0,0, \sqrt{5})
$$

We need not consider $\mathrm{T} \times \Theta$ since it is a subgroup of $\mathrm{O} \times \Theta$.

In $\mathrm{O}\left(\mathrm{D}_{2}\right)$ or $\mathrm{T}\left(\mathrm{D}_{2}\right)$, the element $C_{3} \epsilon$ require that we have instead the linear combination

$$
\begin{aligned}
\Psi= & c\left(x^{4}+\epsilon y^{4}+\epsilon^{2} z^{4}\right) \\
& +a\left(y^{2} z^{2}+\epsilon z^{2} x^{2}+\epsilon^{2} x^{2} y^{2}\right)+b\left(x^{2}+\epsilon y^{2}+\epsilon^{2} z^{2}\right)
\end{aligned}
$$

So far, $c, a, b$ can be complex. This state obviously satisfies $\mathrm{T}\left(\mathrm{D}_{2}\right)$ (after orthogonality to $L=0$ and 2 have been shown). To satisfy the larger group $\mathrm{O}\left(\mathrm{D}_{2}\right), C_{4}^{x} \Theta$ requires that the coefficients $c, a, b$ are all real. [In this case, one can check that elements such as $U_{2}^{\perp x} \Theta$ ( $x \leftrightarrow-x, y \leftrightarrow z$ and then complex conjugate) are also satisfied.] Before we proceed, we note again that the three functions multiplying $c, a, b$ in eq (20) are not linearly independent. Indeed, using $x^{2}+y^{2}+z^{2}=1$ and $1+\epsilon+\epsilon^{2}=0$, we can verify that $\left(x^{4}+\epsilon y^{4}+\epsilon^{2} z^{4}\right)=$ $\left(x^{2}+\epsilon y^{2}+\epsilon^{2} z^{2}\right)+\left(y^{2} z^{2}+\epsilon z^{2} x^{2}+\epsilon^{2} x^{2} y^{2}\right)$. Let us proceed to work with the form with $c=0$. It remains to find $a, b$ to satisfy orthogonality to $L=0$ and $2 .<\Psi>=0$ is trivial (since $\left.1+\epsilon+\epsilon^{2}=0\right) .<\Psi x^{2}>=0$ and $<\Psi y^{2}>=0$ both require $-a+7 b=0\left(<\Psi z^{2}>=0\right.$ is then automatically satisfied), and therefore both $a$ and $b$ can be chosen real. Hence we obtain

$$
\Psi=7\left(y^{2} z^{2}+\epsilon z^{2} x^{2}+\epsilon^{2} x^{2} y^{2}\right)+\left(x^{2}+\epsilon y^{2}+\epsilon^{2} z^{2}\right)
$$

as a state satisfying $\mathrm{O}\left(\mathrm{D}_{2}\right)$ (thus there is no need to consider $\mathrm{T}\left(\mathrm{D}_{2}\right)$ ). In appendix C, we show that, up to a renormalization constant and phase factor, the corresponding spin wavefunction is

$$
\mathrm{C} \equiv(\sqrt{7}, 0, \sqrt{12} i, 0,-\sqrt{10}, 0, \sqrt{12} i, 0, \sqrt{7})
$$

This is our state with $\mathrm{O}\left(\mathrm{D}_{2}\right)$ symmetry. We are calling this state cyclic $(\mathrm{C})$ in analogy with the cyclic state in $L=2$. This completes our consideration of polyhedral groups.

The states with dihedral groups can be found as in $L=2$ and 3 . The state $\mathrm{P}_{4} \equiv$ $(1,0,0,0,0,0,0,0,1)$ has polynomial form $\operatorname{Re}(x+i y)^{4}$ and symmetry

$$
\mathrm{D}_{8}\left(\mathrm{D}_{4}\right) \times \Theta \equiv\left\{E, C_{2}, 2 C_{4}, 4 C_{8} e^{i \pi}, 4 U_{2}, 4 U_{2}^{\prime \prime} e^{i \pi}\right\} \times \Theta
$$


The state $\mathrm{P}_{3} \equiv(0, i, 0,0,0,0,0,-i, 0)$, (related to $(0,1,0,0,0,0,0,1,0)$ by a rotation around $z)$, polynomial form $z \operatorname{Im}(x+i y)^{3}$, has symmetry $\mathrm{D}_{6}\left(\mathrm{D}_{3}\right) \times \Theta($ eq (14) $)$. We need not consider $(0,0,1,0,0,0,1,0,0)$ since then it has the form $\left(7 z^{2}-1\right)\left(x^{2}-y^{2}\right)$ and symmetry $\mathrm{D}_{4}\left(\mathrm{D}_{2}\right) \times \Theta$, and thus is only a subgroup of $\mathrm{D}_{8}\left(\mathrm{D}_{4}\right) \times \Theta$. Also, for the similar reason there is no need to consider $(0,0,0,1,0,1,0,0,0)$ which is proportional to $\left(7 z^{3}-3 z\right) y$ which has symmetry $\mathrm{D}_{2}\left(\mathrm{C}_{2}\right) \times \Theta$. This completes the list of discrete groups.

Summarizing, we found nine inert states: $\mathrm{F}_{4}, \mathrm{~F}_{3}, \mathrm{~F}_{2}, \mathrm{~F}_{1}, \mathrm{P}_{0}$ with continuous symmetries and $\mathrm{O}, \mathrm{C}, \mathrm{P}_{4}, \mathrm{P}_{3}$ with discrete symmetries.

\section{VORTICES}

As an application of our results, we consider topological line defects (vortices) of the spin condensates. The distinct types of vortices have already been considered in [6] for $S=1$ and [16] for the states $\mathrm{F}_{2}, \mathrm{~F}_{1}$ and $\mathrm{C}$ of $S=2$.

The possible types of vortices are determined by the map of a circle in real space to the space of non-equivalent order parameters. We apply the theorem that has been proven in, e.g, [27], which states that $\pi_{1}\left(\mathrm{G}^{\prime} / \mathrm{H}^{\prime}\right)=\mathrm{H}^{\prime} / \mathrm{H}_{0}^{\prime}$. Here, $\mathrm{G}^{\prime}$ is a simply-connected group characterizing the symmetry of the fully symmetric (normal) phase, and $\mathrm{H}^{\prime}$ is the isotropy subgroup (within $\mathrm{G}^{\prime}$ ) of the state under consideration, and $\mathrm{H}_{0}^{\prime}$ is the part of $\mathrm{H}^{\prime}$ that is connected (in the sense of a neighborhood) with the identity $E$. If $\mathrm{H}^{\prime} / \mathrm{H}_{0}^{\prime}$ is abelian, the line defects are simply classified by the elements of this group. Otherwise, the topologically distinct type of vortices are classified by the conjugate classes of this group.

\section{A. $\mathbf{S}=2$}

This has already been treated in [16] (c.f. [17]) except the states $\mathrm{P}_{2}$ and $\mathrm{P}_{0}$. For ease of comparison with $S=3$ and 4 however we shall also repeat the results for the other states below.

For the states $\mathrm{F}_{1,2}$, since a rotation about the $\mathrm{z}$ axis would also generate a gauge transformation, it is simpliest [27] to just consider the symmetry group $\mathrm{SO}(3)$ for the normal state. For $\mathrm{F}_{1}$, a reference state can be taken just as $z(x+i y)$. Then $\mathrm{H}$ contains the identity $E$ alone. To have a simply-connected $\mathrm{G}^{\prime}$, we "lift" $\mathrm{G}=\mathrm{SO}(3)$ to $\mathrm{G}^{\prime}=\mathrm{SU}(2)$, and then $\mathrm{H}^{\prime}$ 
becomes $\mathrm{H}^{\prime}=\{E, Q\}$ where $Q \neq E$ and $Q^{2}=E$. $\mathrm{H}_{0}^{\prime}$ consists of $E$ alone and $\mathrm{H}^{\prime} / \mathrm{H}_{0}^{\prime}=\mathrm{H}^{\prime}$. We thus have two classes of vortices for $F_{1}$.

For $\mathrm{F}_{2}$, we take the reference state $(x+i y)^{2}$. Then $\mathrm{H}=\left\{E, R_{z}(\pi)\right\}$ consisting of two classes. When we lift from $\mathrm{G}=\mathrm{SO}(3)$ to $\mathrm{G}^{\prime}=\mathrm{SU}(2)$, each element in $\mathrm{H}$ is doubled. Thus there are four classes of vortices.

For $\mathrm{P}_{0}$, we choose the reference state $\left(2 z^{2}-x^{2}-y^{2}\right)$ and take $\mathrm{G}=\mathrm{SO}(3) \times \mathrm{U}(1)$. Then $\mathrm{H}=\mathrm{H}_{0}^{\mathrm{e}}=\left\{R_{z}(\alpha), U_{2}^{\gamma}\right\}$ (see eq (1) consisting of two classes. We need however a simplyconnected $\mathrm{G}^{\prime}$, which can be taken to be $\mathrm{G}^{\prime}=\mathrm{SU}(2) \times T_{\phi}$, where $T_{\phi}$ is the translational group for the phase $\phi$. Then $\mathrm{H}^{\prime}=\left\{u(\hat{z}, \alpha) T_{2 n \pi}, u(\hat{\gamma}, \pm \pi) T_{2 n \pi}\right\}$. Here, we have used the representation $u(\hat{n}, \alpha)=\cos \frac{\alpha}{2}+i \hat{n} \cdot \sigma \sin \frac{\alpha}{2}$ in terms of Pauli matrices for the rotations in $\mathrm{SU}(2)$. $\mathrm{H}_{0}^{\prime}=\{u(\hat{z}, \alpha)\}$, and thus $\mathrm{H}^{\prime} / \mathrm{H}_{0}^{\prime}$ consists of the elements (cosets) $\ldots,\left\{u(\hat{z}, \alpha) T_{-2 \pi}\right\},\{u(\hat{z}, \alpha)\}$, $\left\{u(\hat{z}, \alpha) T_{+2 \pi}\right\}, \ldots,\left\{u(\hat{\gamma}, \pm \pi) T_{2 \pi}\right\}, \ldots$ Since a product between $u\left(\hat{z}, \alpha_{1}\right)$ and $u\left(\hat{z}, \alpha_{2}\right)$ or between $u\left(\hat{\gamma}_{1}, \pm \pi\right)$ and $u\left(\hat{\gamma}_{2}, \pm \pi\right)$ both yield $u(\hat{z}, \alpha)$ for some $\alpha$; and the product between $u(\hat{z})$ and $u(\hat{\gamma}, \pm \pi)$ yield another element $u\left(\hat{\gamma}^{\prime}, \mp \pi\right), \mathrm{H}^{\prime} / \mathrm{H}_{0}^{\prime}$ is isomorphic to $\mathrm{Z}_{2} \times \mathrm{Z}$, where we can assign the integer 0 to $\{u(\hat{z}, \alpha)\}$ and 1 to $\{u(\hat{\gamma}, \pm \pi)\}$ for the group $\mathrm{Z}_{2}$ and the circulation number $n$ for the group Z. Note this is different [16] from the polar state $\mathrm{P}_{0}$ for spin $S=1$. The source of difference is that the isotropy group for the state $\mathrm{P}_{0}$ is $\mathrm{H}_{0}^{\mathrm{e}}$ for $S=2$ but $\mathrm{H}_{0}^{\circ}$ for $S=1$.

The cyclic state $\mathrm{C}$ has been treated in [16] using the language of the spin-wavefunction and its rotational properties. Here the symmetries we obtained before becomes handy. If $\mathrm{G}=\mathrm{SO}(3) \times \mathrm{U}(1) \times \Theta$, we have already found $\mathrm{H}=\mathrm{O}\left(\mathrm{D}_{2}\right)$ (eq (5)). To consider the vortices, we only need to consider instead $\mathrm{G}=\mathrm{SO}(3) \times \mathrm{U}(1)$, and then we obtain $\mathrm{H}=$ $\left\{E, 3 C_{2}, 4 C_{3} \epsilon, 4 C_{3}^{2} \epsilon^{2}\right\}$. This group is isomorphic to the tetrahedral group with $\mathrm{T}=$ $\left\{E, 3 C_{2}, 4 C_{3}, 4 C_{3}^{2}\right\}$ which has four conjugate classes. The double group $\mathrm{T}^{\prime}$ corresponding $\mathrm{T}$ has seven classes [28], $\{E\},\{Q\},\left\{3 C_{2}, 3 C_{2} Q\right\},\left\{4 C_{3}\right\},\left\{4 C_{3} Q\right\},\left\{4 C_{3}^{2}\right\},\left\{4 C_{3}^{2} Q\right\}$, as each class in $\mathrm{T}$ is doubled except the one $\left(\left\{3 C_{2}\right\}\right)$ which consists of bilateral two-fold axes [26]. When $\mathrm{G}=\mathrm{SO}(3) \times \mathrm{U}(1)$ is lifted to the $\mathrm{G}^{\prime}=\mathrm{SU}(2) \times T_{\phi}$, we get $\mathrm{H}_{0}^{\prime}=\{E\}, \mathrm{H}^{\prime} / \mathrm{H}_{0}^{\prime}=\mathrm{H}^{\prime}$ where the group H' has classes $\left\{E T_{2 n \pi}\right\},\left\{Q T_{2 n \pi}\right\},\left\{3 C_{2} T_{2 n \pi}, 3 C_{2} Q T_{2 n \pi}\right\},\left\{4 C_{3} T_{\left(2 n+\frac{2}{3}\right) \pi}\right\}$, $\left\{4 C_{3} Q T_{\left(2 n+\frac{2}{3}\right) \pi}\right\},\left\{4 C_{3}^{2} T_{\left(2 n+\frac{4}{3}\right) \pi}\right\},\left\{4 C_{3}^{2} Q T_{\left(2 n+\frac{4}{3}\right) \pi}\right\}$, where $n$ is an integer. Thus the vortices are divided into seven types in additional to the circulation number $n$. Note however due to the presence of $\epsilon=e^{i 2 \pi / 3}$ factors in $\mathrm{H}$ and hence $\mathrm{H}^{\prime}, \mathrm{H}^{\prime} / \mathrm{H}_{0}^{\prime}$ is not isomorphic to the product of the double group $\mathrm{T}^{\prime}$ times the discrete translational group $T_{2 n \pi}$. 
The polar state $\mathrm{P}_{2}$ with discrete symmetry can be treated similarly. If $\mathrm{G}=\mathrm{SO}(3) \times \mathrm{U}(1)$, then $\mathrm{H}$ is simply $\mathrm{D}_{4}\left(\mathrm{D}_{2}\right)=\left\{E, C_{2}, 2 U_{2}, 2 C_{4} e^{i \pi}, 2 U_{2}^{\prime} e^{i \pi}\right\}$ isomorphic to $\mathrm{D}_{4}=\left\{E, 2 C_{2}, 2 U_{2}, 2 C_{4}, 2 U_{2}^{\prime}\right\}$ with five classes. The double group $\mathrm{D}_{4}^{\prime}$ corresponding to $\mathrm{D}_{4}$ consists of seven classes [28]: $\{E\},\{Q\},\left\{C_{2}, C_{2} Q\right\},\left\{2 U_{2}, 2 U_{2} Q\right\},\left\{C_{4}, C_{4}^{3} Q\right\},\left\{C_{4}^{3}, C_{4} Q\right\},\left\{2 U_{2}^{\prime}, 2 U_{2}^{\prime} Q\right\} . \quad$ When we lift to the connected group $\mathrm{G}^{\prime}=\mathrm{SU}(2) \times T_{\phi}$, we obtain $\mathrm{H}_{0}^{\prime}=\{E\}$, and $\mathrm{H}^{\prime} / \mathrm{H}_{0}^{\prime}=\mathrm{H}^{\prime}$ consists of the following classes: $\left\{E T_{2 n \pi}\right\},\left\{Q T_{2 n \pi}\right\},\left\{C_{2} T_{2 n \pi}, C_{2} Q T_{2 n \pi}\right\},\left\{2 U_{2} T_{2 n \pi}, 2 U_{2} Q T_{2 n \pi}\right\}$, $\left\{C_{4} T_{2 n \pi}, C_{4}^{3} Q T_{2 n \pi}\right\}, \quad\left\{C_{4}^{3} T_{(2 n+1) \pi}, C_{4} Q T_{(2 n+1) \pi}\right\}, \quad\left\{2 U_{2}^{\prime} T_{(2 n+1) \pi}, 2 U_{2}^{\prime} Q T_{(2 n+1) \pi}\right\}$. There are seven vortex types in additional to the circulation numbers $n$.

\section{B. $S=3$}

For the state $\mathrm{F}_{3}$, it is again simpliest just to use $\mathrm{SO}(3)$ and the reference state $(x+i y)^{3}$. $\mathrm{H}$ consists of three elements, $E, R_{z}(2 \pi / 3), R_{z}(4 \pi / 3)$. The corresponding double group $\mathrm{H}^{\prime}$ has six elements. There are six distinct classes of vortices.

$\mathrm{F}_{2}$ and $\mathrm{F}_{1}$ has the same corresponding $\mathrm{H}$ as their $S=2$ counterpart. The distinct classes of vortices are again four and two respectively.

For the polar state $\mathrm{P}_{0}$, we choose the reference state $\left(5 z^{3}-3 z\right)$ and take $\mathrm{G}=\mathrm{SO}(3)$ $\times \mathrm{U}(1)$. Then $\mathrm{H}=\left\{R_{z}(\alpha), U_{2}^{\gamma} e^{i \pi}\right\}$ (see eq (2) ) consisting of two classes. When using the connected $\mathrm{G}^{\prime}=\mathrm{SU}(2) \times T_{\phi}, \mathrm{H}^{\prime}=\left\{u(\hat{z}, \alpha) T_{2 n \pi}, u(\hat{\gamma}, \pm \pi) T_{(2 n+1) \pi}\right\} . \mathrm{H}_{0}^{\prime}=\{u(\hat{z}, \alpha)\}$, and thus $\mathrm{H}^{\prime} / \mathrm{H}_{0}^{\prime}$ consists of the elements (cosets) .., $\left\{u(\hat{z}, \alpha) T_{-2 \pi}\right\},\{u(\hat{z}, \alpha)\},\left\{u(\hat{z}, \alpha) T_{+2 \pi}\right\}, \ldots$, $\left\{u(\hat{\gamma}, \pm \pi) T_{\pi}\right\}, \ldots$, Note now that the cosets with $u(\hat{\gamma}, \pm \pi)$ are associated with translation of phase of odd integral multiple of $\pi$. Again noting the properties of the products of $u(\hat{n}, \alpha)$ discussed in $S=2$, we find that $\mathrm{H}^{\prime} / \mathrm{H}_{0}^{\prime}$ is isomorphic to $\mathrm{Z}$, as it is the case [16] for the state $\mathrm{P}_{0}$ belong to $S=1$.

For the state $\mathrm{D}$, when we just use $\mathrm{G}=\mathrm{SO}(3) \times \mathrm{U}(1)$ then $\mathrm{H}=\mathrm{O}(\mathrm{T})=$ $\left\{E, 8 C_{3}, 3 C_{2}, 6 U_{2} e^{i \pi}, 6 C_{4} e^{i \pi}\right\}$ is isomorphic to $O=\left\{E, 8 C_{3}, 3 C_{2}, 6 U_{2}, 6 C_{4}\right\}$ with thus five classes. The double group $\mathrm{O}^{\prime}$ corresponding to $\mathrm{O}$ has eight classes, (only $\{E\}$, $\left\{8 C_{3}\right\}$ and $\left\{6 C_{4}\right\}$ are doubled). Vortices can therefore be classified into the following classes: $\left\{E T_{2 n \pi}\right\},\left\{Q T_{2 n \pi}\right\},\left\{4 C_{3}, 4 C_{3}^{2} Q\right\} T_{2 n \pi}, \quad\left\{4 C_{3}^{2}, 4 C_{3} Q\right\} T_{2 n \pi}, \quad\left\{3 C_{2}, 3 C_{2} Q\right\} T_{2 n \pi}$, $\left\{3 C_{4}, 3 C_{4}^{3} Q\right\} T_{(2 n+1) \pi}, \quad\left\{3 C_{4}^{3}, 3 C_{4} Q\right\} T_{(2 n+1) \pi}, \quad\left\{6 U_{2}, 6 U_{2} Q\right\} T_{(2 n+1) \pi}$. There are thus eight classes in additional to the circulation number $n$, and those elements originally associated 
with $e^{i \pi}$ in the group $\mathrm{O}(\mathrm{T})$ are now associated with phase translations $(2 n+1) \pi$.

For the polar state $\mathrm{P}_{3}$, if we just use $\mathrm{G}=\mathrm{SO}(3) \times \mathrm{U}(1)$ then $\mathrm{H}=\mathrm{D}_{6}\left(\mathrm{D}_{3}\right)=$ $\left\{E, C_{2}, 2 C_{3}, 2 C_{6} e^{i \pi}, 3 U_{2}, 3 U_{2}^{\prime} e^{i \pi}\right\}$ was already given in eq (14). This group is isomorphic to $\mathrm{D}_{6}=\left\{E, C_{2}, 2 C_{3}, 2 C_{6}, 3 U_{2}, 3 U_{2}^{\prime}\right\}$ with six classes. The double group $\mathrm{D}_{6}^{\prime}$ corresponding to $\mathrm{D}_{6}$ has nine classes: all classes are doubled except $C_{2}, 3 U_{2}$ and $3 U_{2}^{\prime}$. Vortices are therefore divided to nine classes, in additional to an integer $n$ specifying the circulation. (Those classes that correspond to $C_{6} e^{i \pi}$ and $U_{2}^{\prime} e^{i \pi}$ are associated with phase translations $T_{(2 n+1) \pi}$ rather than $T_{2 n \pi}$ (c.f. the state $\left.\mathrm{D}\right)$.

\section{C. $S=4$}

These can be obtained similarly and so we shall be brief. $\mathrm{F}_{1}, \mathrm{~F}_{2}, \mathrm{~F}_{3}, \mathrm{~F}_{4}$ have respectively two, four, six and eight classes of vortices. $\mathrm{P}_{0}$ has symmetry same as $\mathrm{P}_{0}$ of $S=2$ and thus with the same classes of vortices. A parallel statement applies to the cyclic state C. The state O has eight classes of vortices in additional to the circulation numbers. We note however that all vortices for $\mathrm{O}$ have winding phase factors $2 n \pi$, thus different from the state $\mathrm{D}$ of $S=3$ where the symmetry group is $\mathrm{O}(\mathrm{T})$ instead of $\mathrm{O}$. The state $\mathrm{P}_{4}$ has symmetry group $\mathrm{D}_{8}\left(\mathrm{D}_{4}\right)$. The double group $\mathrm{D}_{8}^{\prime}$ has nine classes. So vortices for $\mathrm{P}_{4}$ has nine classes of vortices in additional to the circulation number, and each class is associated phase translations of $(2 n+1) \pi$ or $2 n \pi$ depending on whether the factor $e^{i \pi}$ appears or not in $\mathrm{D}_{8}\left(\mathrm{D}_{4}\right)$. The state $\mathrm{P}_{3}$ is analogus to that in $S=3$.

\section{CONCLUSIONS}

To conclude, we have listed all inert states for a spin superfluid for $S \leq 4$. These states are always stationary points of any general free energies. We have also given the residual symmetries (hence broken symmetries) of these inert states.

This research is supported by the National Science Council of Taiwan under Grant No. NSC95-2112-M-001-054. 


\section{APPENDIX A: GROUPS WITH FIVE-FOLD SYMMETRY ELEMENTS}

Here we explain why groups containing five-fold symmetry elements $C_{5}$ (without containing the continuous symmetry $\mathrm{C}_{\infty}$ ), in particular the icosahedral group $\mathrm{Y}$, are not involved for inert states with $L \leq 4$. First, it is clear that, since $L \leq 4$, the wavefunction cannot be invariant under $C_{5}$ itself. Next we show that $\mathrm{H}$ cannot contain $\Theta$ itself. For if it did, then it would contain both $C_{5} e^{i \phi_{5}}$ and $C_{5} e^{i \phi_{5}} \Theta$ for some $\phi_{5}$. The latter element can be rewritten as $\Theta C_{5} e^{-i \phi_{5}}$ and hence $\mathrm{H}$ would contain $C_{5}^{2}$, leading to a contradiction. Similarly we can show that $\mathrm{H}$ cannot contain an element of the form $h=C_{5} e^{i \phi_{5}} \Theta$. If $h$ is an element in $\mathrm{H}$, then so must $h^{5}=e^{i \phi_{5}} \Theta$. Thus apart from a gauge transformation, the state $\Psi$ is time-reversal invariant $\left(\Psi^{\prime}=\Psi e^{i \phi_{5} / 2}\right.$ is invariant under $\left.\Theta\right)$. which is again not possible. Therefore $C_{5}$ must be in combination with a gauge transformation only. As seen before, this phase factor must be integral multiple of $2 \pi / 5$. Hence let us consider the element $C_{5} e^{i j 2 \pi / 5}$ where $j$ is an integer. As seen already the non-zero elements of $\zeta_{m}$ must be $\zeta_{j}, \zeta_{j-5}$ etc, and to have discrete but not continuous symmetry at least two of such elements must be non-zero. For $L=1$ and 2 this is not possible. For $L=3$, the only possible pair is $\left(\zeta_{3}, \zeta_{-2}\right)$ if $j=3$, (equivalently $\left(\zeta_{2}, \zeta_{-3}\right)$ ), and for $L=4$ an additional possibility $\left(\zeta_{4}, \zeta_{-1}\right)$ if $j=4$. For these states to be inert one must have other symmetry operations in $\mathrm{H}$ that would impose a relation between these non-zero elements. Clearly a horizontal rotation (and hence groups like $\mathrm{D}_{5}$ ) is not sufficient, and hence one is left with the icosahedral group Y. We recall [25] first that the icosahedral group Y contains, in additional to the five-fold axis pointing along the (12) vertices, three-fold axes perpendicular to the (20) triangular faces and (15) two-fold axes pointing out to the (30) edges of the icosahedron. Recall also that in the group Y, we have $C_{5} C_{3}=C_{2}$, where $C_{3}$ is a three-fold axis normal to the triangle which contains the $C_{5}$ as an vertex, and $C_{2}$ is around the edge of the triangle connected to the mentioned vertex for the $C_{5}$ axis [25]. In the isotropy group $\mathrm{H}$, these two- and three-fold rotations $C_{2}$ and $C_{3}$ must appear as symmetry elements, though they can be combined with again phase factors and/or time reversal symmetry. By similar argument as given already for $C_{5}, C_{3}$ cannot be appear in the form $C_{3} e^{i \phi_{3}} \Theta$ (else the state is again time-reversal invariant), thus the three-fold rotations can appear only via $C_{3} e^{i j^{\prime} 2 \pi / 3}$ for some integer $j^{\prime}$. Therefore now $\mathrm{H}$ must contain the element $C_{5} e^{i j 2 \pi / 5} C_{3} e^{i j^{\prime} 2 \pi / 3}=C_{2} e^{i\left(\frac{j}{5}+\frac{j^{\prime}}{3}\right) 2 \pi} \equiv h_{2}$ and hence $h_{2}^{2}$. But this is possible only if $j=j^{\prime}=0$ since $C_{2}^{2}=1$. Thus we arrive again at the conclusion that $\mathrm{H}$ must 
contain $C_{5}$ itself, which however is impossible for $L \leq 4$. This completes our proof.

\section{APPENDIX B: RELATION BETWEEN STATES D AND D'}

We here show that the state $x y z$, with symmetry $\mathrm{O}(\mathrm{T}) \times \Theta($ eq (4) $)$, is related to

$$
\mathrm{D}^{\prime} \equiv(\sqrt{2}, 0,0, i \sqrt{5}, 0,0, \sqrt{2})
$$

in a new set of orthonormal axes $(\hat{u}, \hat{v}, \hat{w})$. Since $\mathrm{D}^{\prime}$ has explicitly three-fold symmetry along its quantization axis (identified as $\hat{w}$ ) and two-fold axes (up to $e^{i \pi}$ ) perpendicular to it, and $x y z$ has a three-fold axis along the cube diagonal, this suggests that we take $\hat{w}=(\hat{x}+\hat{y}+\hat{z}) / \sqrt{3}, \hat{u}=(-\hat{x}+\hat{y}) / \sqrt{2}$ along one of the $U_{2} e^{i \pi}$ axis, and $\hat{v}=\hat{w} \times \hat{u}$ completing the triad. This transformation between $x, y, z$ and $u, v, w$ is the same as the one used in the $S=2$ cyclic state. We have the relations $x=-\frac{1}{\sqrt{2}} u-\frac{1}{\sqrt{6}} v+\frac{1}{\sqrt{3}} w, y=\frac{1}{\sqrt{2}} u-\frac{1}{\sqrt{6}} v+\frac{1}{\sqrt{3}} w$, and $z=\sqrt{\frac{2}{3}} v+\frac{1}{\sqrt{3}} w$, therefore

$$
x y z=-\sqrt{2} v\left(3 u^{2}-v^{2}\right)+w\left(2 w^{2}-3 u^{2}-3 v^{2}\right) .
$$

On the other hand, we have (in the $u, v, w$ axis) $Y_{3}^{ \pm 3}=\mp \sqrt{\frac{35}{\pi}} \frac{1}{8}(u \pm i v)^{3}, Y_{3}^{0}=\sqrt{\frac{7}{\pi}} \frac{1}{4}\left(5 w^{2}-3\right)$, so state $\mathrm{D}^{\prime}$ is proportional to

$$
\sqrt{\frac{35}{\pi}} \frac{i}{4}\left[-\sqrt{2} v\left(3 u^{2}-v^{2}\right)+w\left(5 w^{2}-3\right)\right] .
$$

Using $u^{2}+v^{2}+w^{2}=1$, we see that state $\mathrm{D}^{\prime}$ is proportional to $i$ times the state $x y z$. We conclude that $\mathrm{D}^{\prime}$ in $(u, v, w)$ axes is the same as $i \mathrm{D}=(0,1,0,0,0,-1,0)$ in $(x, y, z)$ axes.

\section{APPENDIX C: SPIN WAVEFUNCTIONS FOR STATES O AND C OF $S=4$}

We would like to find the spin-wavefunction corresponding to states $\mathrm{O}$ [eq (18)] and C [eq (21)]. We note that both these expressions contain only even powers of $x, y$ and $z$, they must be just linear combinations of $Y_{4}^{4}+Y_{4}^{-4}, Y_{4}^{2}+Y_{4}^{-2}$ and $Y_{4}^{0}$. With $c_{4} \equiv \sqrt{\frac{9}{4 \pi}}$, we have $Y_{4}^{4}+Y_{4}^{-4}=c_{4} \sqrt{\frac{35}{32}}\left(\left(x^{2}+y^{2}\right)^{2}-8 x^{2} y^{2}\right), Y_{4}^{2}+Y_{4}^{-2}=c_{4} \sqrt{\frac{5}{8}}\left(7 z^{2}-1\right)\left(x^{2}-y^{2}\right)$ and $Y_{4}^{0}=c_{4} \frac{1}{8}\left(35 z^{4}-30 z^{2}+3\right)$. For state $\mathrm{O}$, we note further that eq (18) is symmetric under $x^{2} \leftrightarrow y^{2}$, thus $Y_{4}^{2}+Y_{4}^{-2}$ is not involved (as expected since its isotropy group contains the 
element $C_{4}$ ). Noting that $Y_{4}^{0}$ is cylindrically symmetric but $Y_{4}^{4}+Y_{4}^{-4}$ is not, we find easily that the expression eq (18) can be rewritten as

$$
-\frac{1}{c_{4}}\left[Y_{4}^{0}+\sqrt{\frac{5}{14}}\left(Y_{4}^{4}+Y_{4}^{-4}\right)\right]
$$

and hence the spin-wavefunction eq (19).

For the state $\mathrm{C}$, it is more convenient to work with

$$
\Psi^{\prime}=7\left(\epsilon y^{2} z^{2}+\epsilon^{2} z^{2} x^{2}+x^{2} y^{2}\right)+\left(\epsilon x^{2}+\epsilon^{2} y^{2}+z^{2}\right)
$$

Using the definition of $\epsilon$, we get,

$$
\Psi^{\prime}=7\left(-\frac{\left(x^{2}+y^{2}\right) z^{2}}{2}-\frac{\sqrt{3}}{2} i z^{2}\left(x^{2}-y^{2}\right)+x^{2} y^{2}\right)+\left(-\frac{x^{2}+y^{2}}{2}+\frac{\sqrt{3}}{2} i\left(x^{2}-y^{2}\right)+z^{2}\right)
$$

Our task is again simplified by noting that $Y_{4}^{2}+Y_{4}^{-2}$ is the only term that is asymmetric under $x \leftrightarrow y, Y_{4}^{4}+Y_{4}^{-4}$ is the only term that is symmetric under $x \leftrightarrow y$ yet not cylindrically symmetric. We can then find easily

$$
\Psi^{\prime}=\frac{1}{c_{4}}\left[Y_{4}^{0}-\sqrt{\frac{6}{5}} i\left(Y_{4}^{2}+Y_{4}^{-2}\right)-\sqrt{\frac{7}{10}}\left(Y_{4}^{4}+Y_{4}^{-4}\right)\right]
$$

hence the wavefunction is proportional to $\left(-\sqrt{\frac{7}{10}}, 0,-\sqrt{\frac{6}{5}} i, 0,1,0,-\sqrt{\frac{6}{5}} i, 0,-\sqrt{\frac{7}{10}}\right)$ and therefore the expression (22) as shown in text.

[1] J. Stenger, D.M. Stamper-Kurn, H.J. Miesner, A.P. Chikkatur, W. Ketterle, Nature, 396, 345 (1999).

[2] A. Görlitz, T. L. Gustavson, A. E. Leanhardt, R. Löw, A. P. Chikkatur, S. Gupta, S. Inouye, D. E. Pritchard and W. Ketterle, Phys. Rev. Lett. 90, 090401 (2003)

[3] M. S. Chang, C. D. Hamley, M. D. Barrett, J. A. Sauer, K. M. Fortier, W. Zhang, L. You and M. S. Chapman, Phys. Rev. Lett. 92, 140403 (2004); Nature Phys. 1, 111 (2005)

[4] H. Schmaljohann, M. Erhard, J. Kronjäger, M. Kottke, S. van Staa, L. Cacciapuoti, J. J. Arlt, K. Bongs and K. Sengstock, Phys. Rev. Lett. 92040402 (2004)

[5] A. Griesmaier, J. Werner, S. Hensler, J. Stuhler and T. Pfau, Phys. Rev. Lett. 94, 160401 $(2005)$ 
[6] T.L. Ho, Phys. Rev. Lett., 81, 742 (1998).

[7] T. Ohmi and K. Machida, J. Phys. Soc. Jpn., 67, 1822 (1998).

[8] C. V. Ciobanu, S.-K. Yip and T.-L. Ho, Phys. Rev. A, 61, 033607 (2000)

[9] J.-P. Martikainen and K.-A. Suominen, J. Phys. B: At. Mol. Opt. Phys. 34, 4091 (2001)

[10] M. Ueda and M. Koashi, Phys. Rev. A, 65, 063602 (2002)

[11] T. Isoshima and S.-K. Yip, J. Phys. Soc. Japan, 75, 074605 (2006)

[12] R. B. Diener and T.-L. Ho, Phys. Rev. Lett. 96, 190405 (2006)

[13] L. Santos and T. Pfau, Phys. Rev. Lett. 96, 190404 (2006)

[14] T.-L. Ho and S.-K. Yip, Phys. Rev. Lett. 84, 4031 (2000)

[15] N.D. Mermin, Phys. Rev. A, 9, 868 (1974)

[16] H. Mäkelä, Y. Zhang and K.-A. Suominen, J. Phys. A: Math. Gen. 36, 8555 (2003)

[17] R. Barnett, A. Turner and E. Demler, Phys. Rev. Lett. 97, 180412 (2006)

[18] C. Bruder and D. Vollhardt, Phys. Rev. B 34, 131 (1986)

[19] The Superfluid Phases of Helium 3, D. Vollhardt and P. Wölfle, Taylor and Francis, London, 1990.

[20] G. E. Volovik and L. P. Gorkov, Zh. Eksp. Teor. Fiz. 88, 1412 (1985) [ Sov. Phys. JETP 61, $843(1985)]$.

[21] M. Ozaki, K. Machida and T. Ohmi, Prog. Teor. Phys. 74, 221 (1985)

[22] L. Michel, C. R. Acad. Sci. Paris A272, 433 (1971); Rev. Mod. Phys. 52, 617 (1980).

[23] T.-L. Ho and S.-K. Yip, Phys. Rev. Lett. 82, 247 (1999)

[24] P. W. Anderson and P. Morel, Phys. Rev. 123, 1911 (1961)

[25] P. Kramer and R. W. Haase, Group Theory of Icosahedral Quasicrystals, in Introduction to the Mathematics of Quasicrystals, ed. M. V. Jarić, Academic Press, San Diego, 1989.

[26] R. Mirman, Point Groups, Space Groups, Crystals, Molecules, World Scientific, Singapore, 1999.

[27] N. D. Mermin, Rev. Mod. Phys. 51, 591 (1979).

[28] L. D. Landau and E. M. Lifshitz, Quantum Mechanics, third edition, Pergamon, 1999.

[29] Using $<x^{2}>=1 / 3,<x^{4}>=1 / 5,<x^{6}>=1 / 7,<x^{2} z^{2}>=1 / 15,<x^{2} z^{4}>=1 / 35$, $<x^{2} y^{2} z^{2}>=1 / 105$. 